Due to an error in production, this article, which was originally published in the 1 January 1998 issue, Vol. 83, No. 1, pages 415-424, is being republished in its entirety.

\title{
Electrostriction of polarizable materials: Comparison of models with experimental data
}

\author{
Yuri M. Shkel and Daniel J. Klingenberg ${ }^{a)}$ \\ Department of Chemical Engineering and Rheology Research Center, University of Wisconsin, \\ Madison, Wisconsin 53706
}

(Received 28 April 1997; accepted for publication 19 September 1997)

\begin{abstract}
A microscopic model is employed to relate the dielectric tensor to the microstructure of isotropic amorphous or cubic crystalline materials. Electrostriction is characterized by dielectric parameters that describe how the dielectric tensor varies with deformation and the resulting change in the microstructure. These dielectric parameters are obtained for small deformations, and expressed in terms of measurable, macroscopic properties. Unlike previous approaches, we avoid employing the Lorentz cavity approximation to determine the local polarization. Dipole sums are renormalized by treating the dielectric constant of the undeformed material as a known quantity. Predictions agree with our experimental data for different block copolymer films. Further progress in understanding electrostriction and developing improved models requires measuring the deformation dependence of the dielectric tensor for various materials under various conditions (field strength and frequency, deformation frequency, temperature, etc.). (C) 1998 American Institute of Physics.
\end{abstract}

[S0021-8979(98)00601-X]

\section{INTRODUCTION}

Electrostrictive deformation of a dielectric material caused by an electrostatic field ${ }^{1}$ can be exploited in such devices as transducers, actuators, and sensors. Such deformations may be undesirable in other devices where fixed geometries are required or components are susceptible to fatigue. Selecting suitable materials for particular applications requires knowledge of the relationships between material composition and the properties that govern electrostriction.

Electrostriction is commonly treated phenomenologically via a tensor of electrostrictive coefficients, $\gamma_{i j k l}$, linking the strain tensor $u_{i j}\left[=(1 / 2)\left(\partial u_{i} / \partial x_{j}+\partial u_{j} / \partial x_{i}\right)\right.$, where $\mathbf{u}(\mathbf{x})$ is the displacement field] with the electric field $\mathbf{E}^{2,3}$

$$
u_{i j}=\gamma_{i j k l} E_{k} E_{l} .
$$

The ultimate material deformation depends on the mechanical boundary conditions, and thus depends on the elastic properties of the fixtures attached to the material (e.g., the electrodes). The coefficients $\gamma_{i j k l}$ are therefore not strictly material parameters, but rather characteristics of the entire system. This distinction is not important for stiff inorganic materials, where the fixture stiffnesses are negligible compared to that of the material being studied. In a previous article, ${ }^{4}$ we discussed an alternative approach to relate the deformation induced by an electric field to the change in dielectric properties with deformation. The relationship between the dielectric tensor and deformation for an initially isotropic elastic material is $1,5,6$

$$
\epsilon_{i j}=\epsilon^{0} \delta_{i j}+a_{1} u_{i j}+a_{2} u_{k k} \delta_{i j},
$$

\footnotetext{
${ }^{a)}$ Electronic mail:klingen@neep.engr.wisc.edu
}

where $\epsilon^{0}$ is the relative dielectric constant of the nondeformed material. Since the deformation of solids is often very small $\left(u_{i j} \ll 1\right)$, the change in permittivity and deviation from isotropy is typically negligible. On the other hand, the effect of $a_{1}$ and $a_{2}$ on the forces exerted by the electric field, described by Maxwell's stress tensor, 1,5

$$
\sigma_{i j}^{\text {elect. }}=\epsilon_{0} \frac{2 \epsilon^{0}-a_{1}}{2} E_{i} E_{j}-\epsilon_{0} \frac{\epsilon^{0}+a_{2}}{2} E^{2} \delta_{i j},
$$

is not necessarily small. The dielectric parameters $a_{1}$ and $a_{2}$, which are approximately the same magnitude as $\epsilon^{0}$ (see below), have an $O(1)$ effect on the electrostatic stress and are important material parameters that determine deformation in electric fields.

The ability to control electrostrictive deformation requires determining the relationships between material chemistry and the dielectric coefficients $a_{1}$ and $a_{2}$. One technique for measuring these parameters has been described in Ref. 4, but their values have not yet been obtained for many systems. A complementary approach to experimental measurement is to develop models that relate $a_{1}$ and $a_{2}$ to composition. In this article, our objectives are to obtain such a model, and to compare predictions to experimental data on different polymer films.

Developing predictions for $a_{1}$ and $a_{2}$ requires a model for the dielectric properties of a material. One such construct is the Clausius-Mossotti model which is commonly employed to relate chemical composition to the dielectric constant. This model is reasonably accurate for many dielectrics over a wide range of frequencies (including optical 
frequencies, ${ }^{1,7}$ and references therein). In this model, the constituent molecules are assumed to be nonpolar, but polarizable. The induced dipole moment on each molecule is proportional to the local electric field, which is the sum of the applied field and the dipole fields originating from all the other induced dipoles. Determining the latter contribution is problematic since the infinite sum over all dipoles is conditionally convergent. One common method to avoid this problem, originating in 1879 , is to employ the fictitious "Lorentz cavity," "7-9 where a hollow sphere is embedded in a continuum that possesses the dielectric properties that are to be determined. The local field inside the cavity (due to a uniform polarization) is easily calculated. The dielectric constant is obtained by requiring that the polarization in the continuum is equivalent to the total induced dipole moment per unit volume. For polarizable molecules in a vacuum, this leads to the Clausius-Mossotti equation, expressed here as

$$
\epsilon^{0}=\frac{1+2 \alpha n /\left(3 \epsilon_{0}\right)}{1-\alpha n /\left(3 \epsilon_{0}\right)},
$$

where $\alpha$ is the molecular polarizability, $n$ is the number density of molecules, and $\epsilon_{0}=8.8542 \times 10^{-12} \mathrm{~F} / \mathrm{m}$ is the permittivity of free space. The Debye model follows from a similar analysis for freely rotating polarizable molecules with permanent dipole moments of magnitude $p$. The dielectric constant is then given by Eq. (4) with $\alpha$ replaced by $\alpha+p^{2} / 3 k T$.

Stratton ${ }^{1}$ employed Eq. (4) to describe electrostriction in liquids, which can only sustain isotropic deformations. Deformation only influences the number density, $n$, giving $a_{1}=0$ and

$$
a_{2}=-\frac{1}{3}\left(\epsilon^{0}-1\right)\left(\epsilon^{0}+2\right) .
$$

We note that this result will yield the correct change in dielectric constant for isotropic compression or expansion of any material obeying the Clausius-Mossotti or Debye models.

Anderson ${ }^{10}$ and $\mathrm{Kloos}^{11}$ employed the Lorentz cavity to investigate electrostriction in nonpolar, polarizable solids that can support arbitrary deformations. The materials were treated as crystalline (cubic ${ }^{10}$ and body-centered cubic $^{11}$ ), considered to be models for isotropic media. In each case, the local electric field was separated into three contributions: the applied field, a contribution arising from nearby induced dipoles whose effects were determined directly, and a third contribution arising from distant dipoles whose effects were approximated by that of a Lorentz cavity surrounding the nearby dipoles. Such an approach seems natural given the success of the Clausius-Mossotti model. However, the local field is sensitive to the dipole positions ${ }^{11,12}$ and the cavity shape. ${ }^{13}$ Thus lattice models may not be appropriate for analyzing the dielectric properties of amorphous microstructures. Furthermore, in order to describe electrostriction, the displacement of the nearby dipoles as well as the deformation of the Lorentz cavity must be specified.

Models for electrostriction of amorphous materials have not been previously available, although such systems have many practical applications. ${ }^{14-21}$ In this article, we present a method for describing electrostriction of arbitrary isotropic microstructures (amorphous or cubic crystalline). We relate the dielectric parameters $a_{1}$ and $a_{2}$ to the microstructure without employing the Lorentz cavity formalism. The medium is treated as nonpolar, polarizable entities in a dielectric continuum. This corresponds to the system described by the Clausius-Mossotti model when the dielectric constant of the continuum is equal to one. The dielectric tensor of the medium is related to the arrangement of the polarizable inclusions via a self-consistent mean-field approach. ${ }^{7,13,22}$ Deformation may in principle affect the dielectric tensor in a number of ways, for example, by altering the continuous phase dielectric properties, the inclusion polarizability, or the distribution of inclusions. As in Refs. 10 and 11, we consider only the effect of deformation on the microstructure. We assume that the inclusions displace affinely with the macroscopic deformation, as in Refs. 10 and 11, although the same approach can be applied for other microstructural dynamics (this is discussed further in the following sections). The inclusion microstructure is described by singlet and pair probability distribution functions. The singlet and pair distribution functions are related to the deformation through conservation equations obtained from a general $N$-particle conservation equation. ${ }^{23,24}$ The electrostriction parameters are obtained directly by comparing the mean-field expression for the deformation-dependent permittivity tensor with Eq. (2). The general formalism developed here is illustrated for a cubic lattice and a homogeneous, isotropic, disordered microstructure. Calculations employing this formalism illustrate that the dielectric parameters $a_{1}$ and $a_{2}$ differ significantly between amorphous and cubic microstructures.

The method for obtaining the electrostriction parameters is presented in the following section. In Sec. II A, the dielectric tensor is related to the net polarization in the medium. The self-consistent mean-field approach relating the dielectric tensor to the microstructure is presented in Sec. II B. Section II C illustrates the general approach by dealing with electrostriction of a cubic lattice. Convergence problems with dipole sums are avoided by treating the dielectric constant of the undeformed material as a known quantity. In Sec. II D, the evolution of an amorphous microstructure with deformation is determined through conservation equations linking the distribution functions with the macroscopic strain field. The deformation-dependent distribution functions are combined with the mean-field result for the dielectric tensor to obtain the dielectric parameters in Sec. II E. This is the main result of this article. We find that an amorphous medium consisting of two nonelectrostrictive phases is itself electrostrictive, arising from two different effects: the change in inclusion number density and the appearance of anisotropy in the pair distribution function. In Sec. III, we summarize the main features and assumptions of the model, and briefly discuss how these assumptions may be relaxed to describe additional features. Predictions are also compared with previous models. In Sec. IV, we present experimental data for three solid block copolymer films. Measured values of $a_{1}+a_{2}$ compare very well with predictions. The main conclusions of this article are summarized in Sec. V. 


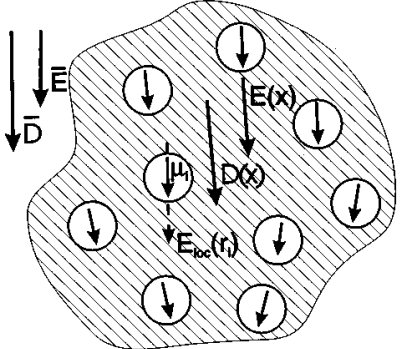

FIG. 1. Schematic diagram depicting the heterogeneous medium. $\overline{\mathbf{E}}$ and $\overline{\mathbf{D}}$ are the macroscopic (volume-averaged) electric field and dielectric displacement, respectively, $\mathbf{E}(\mathbf{x})$ and $\mathbf{D}(\mathbf{x})$ are the position-dependent microscopic fields (defined in both phases), $\boldsymbol{\mu}_{\mathbf{i}}$ is the excess dipole moment of inclusion $i$, and $\mathbf{E}_{\mathrm{loc}}\left(\mathbf{r}_{i}\right)$ is the local electric field that would exist at $\mathbf{r}_{i}$ in the absence of inclusion $i$.

\section{EFFECT OF DEFORMATION ON MATERIAL POLARIZATION}

\section{A. Polarization of a heterogeneous material}

We consider a material consisting of polarizable entities dispersed in a dielectric matrix with dielectric constant $\epsilon_{c}$ (see Fig. 1). The medium is assumed to be dielectrically isotropic prior to deformation. The macroscopic dielectric displacement $\overline{\mathbf{D}}$ and electric field $\overline{\mathbf{E}}$ (i.e., averaged over a volume containing many inclusions) are connected with the microscopic dielectric displacement $\mathbf{D}(\mathbf{x})$ and the electric field $\mathbf{E}(\mathbf{x})$ by the integral

$$
\overline{\mathbf{D}}-\epsilon_{0} \epsilon_{c} \overline{\mathbf{E}}=\frac{1}{V} \int_{V}\left(\mathbf{D}(\mathbf{x})-\epsilon_{0} \epsilon_{c} \mathbf{E}(\mathbf{x})\right) d \mathbf{x} .
$$

The integrand is zero everywhere except within the polarizable inclusions, giving

$$
\begin{aligned}
\boldsymbol{\epsilon}_{0}\left(\boldsymbol{\epsilon}-\boldsymbol{\epsilon}_{c} \boldsymbol{\delta}\right) \cdot \overline{\mathbf{E}} & =\frac{1}{V} \sum_{i=1}^{N} \int_{V_{i}}\left[\mathbf{P}(\mathbf{x})-\epsilon_{0}\left(\boldsymbol{\epsilon}_{c}-1\right) \mathbf{E}(\mathbf{x})\right] d \mathbf{x} \\
& =\frac{1}{V} \sum_{i=1}^{N} \boldsymbol{\mu}_{i}=\mathbf{m},
\end{aligned}
$$

where $\boldsymbol{\mu}_{i}$ is the dipole moment due to inclusion $i$ in excess of the polarizable medium, $N$ is the number of inclusions in the volume $V$, and $\mathbf{m}$ is the polarization $\left(\mathrm{C} / \mathrm{m}^{2}\right)$ in excess of that in the dielectric matrix. The problem of determining the "effective medium" dielectric tensor $\epsilon_{i j}$ for heterogeneous materials thus reduces to determining the excess polarization for a given macroscopic field $\overline{\mathbf{E}}$. This is described in the next section.

Analysis is simplified by choosing a coordinate system codirected with the principal axes of the macroscopic strain tensor $u_{i j}$ (which will be taken as uniform in the volume under consideration). In this coordinate system, $u_{i j}$, as well as $\epsilon_{i j}$ [Eq. (2)], have only diagonal terms, and the polarization in an arbitrary field can be decomposed into three components:

$$
\begin{aligned}
& \overline{\mathbf{D}}=\bar{D}_{1} \mathbf{e}_{1}+\bar{D}_{2} \mathbf{e}_{2}+\bar{D}_{3} \mathbf{e}_{3}, \\
& \bar{D}_{\xi}=\epsilon_{0} \epsilon_{\xi} \overline{\mathbf{E}} \cdot \mathbf{e}_{\xi},
\end{aligned}
$$

$$
\epsilon_{\xi}=\epsilon^{0}+a_{1} u_{\xi \xi}+a_{2} u_{k k},
$$

where $\mathbf{e}_{\xi}(\xi=1,2,3)$ are the eigenvectors of $u_{i j}$ and $\epsilon_{i j}$. Assuming that the coefficients $\epsilon^{0}, a_{1}$, and $a_{2}$ do not depend on the electric field magnitude, the problem is reduced to considering the material's polarization in an electric field codirected with one of the eigenvectors of the strain tensor.

\section{B. Mean field approach}

The dipole moment of a single inclusion $i, \boldsymbol{\mu}_{i}$, is proportional to the local electric field $\mathbf{E}_{\mathrm{loc}}\left(\mathbf{r}_{i}\right)$, which is the sum of the applied field $\overline{\mathbf{E}}$ and the disturbance fields produced by the other inclusions. Ignoring quadrupole and higher-order disturbance fields,

$$
\boldsymbol{\mu}_{i}=\alpha \mathbf{E}_{\mathrm{loc}}\left(\mathbf{r}_{i}\right)=\alpha \overline{\mathbf{E}}-\alpha \sum_{j \neq i}^{N} \mathbf{T}\left(\mathbf{r}_{i j}\right) \cdot \boldsymbol{\mu}_{j},
$$

where $\alpha$ is the polarizability and

$$
\mathbf{T}\left(\mathbf{r}_{i j}\right)=\frac{1}{4 \pi \epsilon_{0} \epsilon_{c}}\left[\frac{\boldsymbol{\delta}}{r_{i j}^{3}}-3 \frac{\mathbf{r}_{i j} \mathbf{r}_{i j}}{r_{i j}^{5}}\right], \quad \mathbf{r}_{i j}=\mathbf{r}_{j}-\mathbf{r}_{i}
$$

is the dipole interaction tensor describing the disturbance field at $\mathbf{r}_{i}$ due to a dipole at $\mathbf{r}_{j}$. Neglecting the higher-order terms in Eq. (10) simplifies the problem, but restricts the approach to weakly polarizable inclusions. If Eq. (10) is applied to a dilute suspension of polarizable spheres (dielectric constant $\epsilon_{p}$, radius $r_{0}$ ), the polarizability is $\alpha=4 \pi \epsilon_{0} \epsilon_{c} r_{0}^{3}$ $\times\left(\epsilon_{p}-\epsilon_{c}\right) /\left(\epsilon_{p}+2 \epsilon_{c}\right)$; for conducting spheres in an insulating medium, $\alpha=4 \pi \epsilon_{0} \epsilon_{c} r_{0}^{3}$.

The polarization caused by an electric field applied in the $\xi$ direction is

$$
\begin{aligned}
m_{\xi} & =\alpha n \bar{E}_{\xi}-\frac{\alpha}{V} \sum_{i=1}^{N} \sum_{j \neq i}^{N} \mathbf{e}_{\xi} \cdot \mathbf{T}\left(\mathbf{r}_{i j}\right) \cdot \boldsymbol{\mu}_{j} \\
& =\alpha n \bar{E}_{\xi}-\alpha n \sum_{j \neq i}^{N}\left\langle\mathbf{e}_{\xi} \cdot \mathbf{T}\left(\mathbf{r}_{i j}\right) \cdot \boldsymbol{\mu}_{j}\right\rangle,
\end{aligned}
$$

where $n=N / V$, and the average over all reference inclusions $i$ is equated with the ensemble average over all realizations. We now employ the "mean-field" assumption,

$$
\left\langle\mathbf{e}_{\xi} \cdot \mathbf{T}\left(\mathbf{r}_{i j}\right) \cdot \boldsymbol{\mu}_{j}\right\rangle=\mu_{\xi}\left\langle\mathbf{e}_{\xi} \cdot \mathbf{T}\left(\mathbf{r}_{i j}\right) \cdot \mathbf{e}_{\xi}\right\rangle,
$$

where $\mu_{\xi}=\left\langle\boldsymbol{\mu}_{j}\right\rangle \cdot \mathbf{e}_{\xi}=m_{\xi} / n$. This states that each inclusion contributes equivalently to the local field at some point, depending only on its relative position. The last term in Eq. 12 may be expressed in terms of a distribution function,

$$
\begin{aligned}
\Lambda_{\xi} & \equiv \sum_{j \neq i}^{N}\left\langle\mathbf{e}_{\xi} \cdot \mathbf{T}\left(\mathbf{r}_{i j}\right) \cdot \mathbf{e}_{\xi}\right\rangle, \\
& \simeq \int_{V}\left(\mathbf{e}_{\xi} \cdot \mathbf{T}(\mathbf{r}) \cdot \mathbf{e}_{\xi}\right) \mathscr{P}(\mathbf{r} \mid \mathbf{0}) d \mathbf{r},
\end{aligned}
$$

where $\mathscr{P}(\mathbf{r} \mid \mathbf{0})$ is the conditional probability density for a second inclusion located at $\mathbf{r}$ given that one is also located at the origin. The mean-field assumptions allow us to resolve Eq. (12) to express the total excess polarization self-consistently, 


$$
m_{\xi}=\frac{\alpha n}{1+\alpha \Lambda_{\xi}} \bar{E}_{\xi} .
$$

Substituting this expression for $m_{\xi}$ into Eq. (7) gives the component $\epsilon_{\xi}$ of the dielectric tensor,

$$
\epsilon_{\xi}=\epsilon_{c}+\frac{\alpha n}{\epsilon_{0}\left(1+\alpha \Lambda_{\xi}\right)} .
$$

Similar expressions have been obtained by others. ${ }^{7,11,22}$ Ignoring quadrupole and higher-order electrostatic interactions in Eq. (10) is strictly valid only for dilute suspensions of weakly polarizable inclusions. However, the mean-field analysis approximately describes the multibody effect of all the inclusions increasing the field magnitude "seen" by each individual one. ${ }^{13}$ As shown below, this model recovers the Clausius-Mossotti (or Maxwell) relation for the effective dielectric constant of a suspension, which has been shown to work reasonably well for a wide range of polarizabilities and concentrations. $^{22}$

Since electrostriction is the effect of deformation on $\epsilon_{\xi}$, one need only consider how $u_{i j}$ can influence terms on the right-hand side of Eq. (17). Each of the terms $\alpha, n, \epsilon_{c}$ and $\Lambda_{\xi}$ may depend on $u_{i j}$. In the next section, we analyze the effect of deformation directly on $n$ and $\Lambda_{\xi}$ for a cubic lattice. In the following section, we determine the effect of deformation on $n$ and $\mathscr{P}(\mathbf{r} \mid \mathbf{0})$ for arbitrary and initially isotropic structures. The effect of the deformation of $\alpha$ and $\epsilon_{c}$ will be considered briefly in the discussion.

\section{Electrostriction of a cubic lattice}

We consider first a material composed of polarizable inclusions arranged on a cubic lattice. The microstructure is assumed to deform affinely, and the variation in permittivity with deformation is attributed only to the changes in the dipole number density and the local field. Anderson ${ }^{10}$ considered the strain dependence of this system by employing the Lorentz cavity approach. In this section, we consider electrostriction of a cubic lattice by employing another approach that avoids the Lorentz cavity. The approach illustrated here is then employed in the following section to analyze electrostriction of amorphous structures.

Calculating $\Lambda_{\xi}$ involves significant theoretical difficulties, even for an undeformed cubic lattice where the material structure is known exactly. For an undeformed cubic lattice, Eq. (14) becomes

$$
\Lambda^{0}=\frac{1}{4 \pi \epsilon_{0} \epsilon_{c} a^{3}} \sum \frac{i^{2}+j^{2}-2 k^{2}}{\left(i^{2}+j^{2}+k^{2}\right)^{5 / 2}},
$$

where the $a$ is the cubic lattice constant $\left(n^{0}=1 / a^{3}\right)$ and the sum is over $N^{0}$ dipoles within a control volume $V$. The superscript ${ }^{0}$ refers to quantities in the nondeformed state. This sum can be evaluated for any specific volume, with a finite value obtained when the volume is expanded to infinity. However, the result is not unique and depends on the volume shape; such a sum is conditionally convergent. Rayleigh ${ }^{25}$ (in 1892) first encountered this difficulty, noting that the summation [Eq. (18)] over a cubic volume gives zero for cubic arrays. The Lorentz cavity approach eliminates this problem

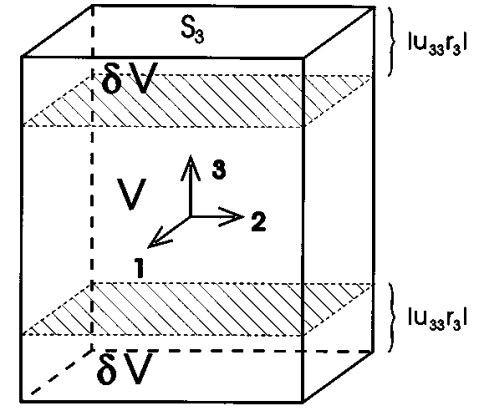

FIG. 2. Schematic diagram depicting the control volume $V$ and shell volume $\delta V$ (illustrated here for compression along the $\mathbf{e}_{3}$ axis). The volume $V$ contains $N^{0}=V / a^{3}$ dipoles prior to deformation and $N=N^{0}+N_{S}$ dipoles after deformation, where $N_{S}=\delta V / a^{3}$ is the number of dipoles that enter $V$ upon deformation.

by considering the dipoles outside of the volume $V$ as a continuous medium. The field from charges on the bounding walls corrects for the effect of the volume shape.

The convergence problem can also be solved by renormalization of Eq. (12), so that an expression similar to Eq. (14) can be obtained without ending up with a conditionally convergent sum. This approach has been successfully applied to suspension rheology, ${ }^{26}$ conduction through a random suspension of spheres, ${ }^{13}$ and to a microscopic model of electrorheology. ${ }^{22}$ Convergence in each of these problems is obtained by noting that the macroscopic field $\left(\bar{E}_{\xi}\right.$ in our case) is known, and can also be expressed in terms of the field distortions due to each dipole, which forms a sum similar to Eq. (14).

We have found that convergence difficulties can be avoided with a different approach. The sum $\Lambda^{0}$ is assumed to be a known quantity, expressed simply as a function of the dielectric constant of the undeformed material, $\epsilon^{0}$, via Eq. (17). We find that $\Lambda_{\xi}$ for a deformed material can then be expressed as a function of $\Lambda^{0}$ and other absolutely convergent summations. Thus convergence problems reside only in the calculation of $\Lambda^{0}$ for the undeformed state. $\Lambda^{0}$ can in principle be determed via direct measurement of the dielectric constant $\epsilon^{0}$, or calculated either by renormalization or Lorentz cavity approaches; both calculations give the same value, $\Lambda^{0}=1 /\left(3 a^{3} \epsilon_{0} \epsilon_{c}\right)$.

The quantity $\Lambda_{\xi}$ is obtained by the direct summation of Eq. (14) over a fixed control volume $V$,

$$
\Lambda_{\xi}=\sum_{\mathbf{r}_{\alpha} \in V} \mathbf{e}_{\xi} \cdot \mathbf{T}\left(\mathbf{r}_{\alpha}\right) \cdot \mathbf{e}_{\xi}
$$

where $\mathbf{r}_{\alpha}$ is the position of inclusion $\alpha$ after deformation (initially at $\mathbf{r}_{\alpha}^{0}$ ). Prior to deformation, the control volume contains $N^{0}=V / a^{3}$ inclusions. As a result of deformation, inclusions within a shell of volume $\delta V$ near the bounding surface of $V$ will enter (or leave) the control volume, as illustrated in Fig. 2. Distinguishing between those inclusions originally in $V$ from those that enter or leave $V$, Eq. (19) may be rewritten 


$$
\Lambda_{\xi}=\sum_{\mathbf{r}_{\alpha}^{0} \in V} \mathbf{e}_{\xi} \cdot \mathbf{T}\left(\mathbf{r}_{\boldsymbol{\alpha}}\right) \cdot \mathbf{e}_{\xi} \pm \sum_{\mathbf{r}_{\alpha}^{0} \in \delta V} \mathbf{e}_{\xi} \cdot \mathbf{T}\left(\mathbf{r}_{\boldsymbol{\alpha}}\right) \cdot \mathbf{e}_{\xi},
$$

where the contributions are added or subtracted depending on whether the inclusions enter or leave $V$ during the deformation. Since $\delta V / V \propto\|u\|$, this may be rewritten for small deformations as

$$
\begin{aligned}
\Lambda_{\xi}= & \sum_{\mathbf{r}_{\alpha}^{0} \in V} \mathbf{e}_{\xi} \cdot \mathbf{T}\left(\mathbf{r}_{\alpha}^{0}\right) \cdot \mathbf{e}_{\xi}+\left.\sum_{\mathbf{r}_{\alpha}^{0} \in V} \mathbf{r} \cdot \mathbf{u} \cdot \nabla\left[\mathbf{e}_{\xi} \cdot \mathbf{T}(\mathbf{r}) \cdot \mathbf{e}_{\xi}\right]\right|_{\mathbf{r}_{\alpha}^{0}} \\
& \pm \sum_{\mathbf{r}_{\alpha}^{0} \in \delta V} \mathbf{e}_{\xi} \cdot \mathbf{T}\left(\mathbf{r}_{\alpha}^{0}\right) \cdot \mathbf{e}_{\xi}+\mathscr{Q}\left(\|\mathbf{u}\|^{2}\right)
\end{aligned}
$$

For isotropic deformations, Eq. (21) reduces to $\Lambda_{\xi}=\Lambda^{0}\left(1-u_{k k}\right)$; this result can also be obtained directly from Eq. (18) after substituting $a^{3}\left(1-u_{k k}\right)$ for $a^{3}$.

We now consider the volume $V$ as a right parallelepiped of dimensions $\left(2 N_{1}+1\right) a \times\left(2 N_{2}+1\right) a \times\left(2 N_{3}+1\right) a$ with the edges parallel to the lattice vectors. Let the applied electric field be codirected with $\mathbf{e}_{3}$. The number of inclusions that pass into $V$ per unit area through each of the planar bounding surfaces at $x= \pm N_{1} a$ is given by $-u_{11} N_{1}$; the number per unit area passing into $V$ through the surfaces at $y= \pm N_{2} a$ and $z= \pm N_{3} a$, are given by $-u_{22} N_{2}$ and $-u_{33} N_{3}$, respectively. Expanding the arguments in the last term about the nearest bounding surface, and separating the contribution from the isotropic part of the deformation, Eq. (21) becomes

$$
\Lambda_{\xi}=\Lambda^{0}\left(1-u_{k k}\right)+\frac{1}{\epsilon_{0} \epsilon_{c} a^{3}}\left[u_{11} \Sigma_{1}+u_{22} \Sigma_{2}+u_{33} \Sigma_{3}\right],
$$

where $\Sigma_{1}, \Sigma_{2}$, and $\Sigma_{3}$ are given by

$$
\begin{aligned}
\Sigma_{1}= & \frac{1}{4 \pi}\left\{\sum _ { i = - N _ { 1 } } ^ { N _ { 1 } } \sum _ { j = - N _ { 2 } } ^ { N _ { 2 } } \sum _ { k = - N _ { 3 } } ^ { N _ { 3 } } \left[\frac{i^{2}+j^{2}-2 k^{2}}{\left(i^{2}+j^{2}+k^{2}\right)^{5 / 2}}\right.\right. \\
& \left.-\frac{3 i^{2}\left(i^{2}+j^{2}-4 k^{2}\right)}{\left(i^{2}+j^{2}+k^{2}\right)^{7 / 2}}\right] \\
& \left.-2 N_{1} \sum_{j=-N_{2}}^{N_{2}} \sum_{k=-N_{3}}^{N_{3}} \frac{N_{1}^{2}+j^{2}-2 k^{2}}{\left(N_{1}^{2}+j^{2}+k^{2}\right)^{5 / 2}}\right\}, \\
\Sigma_{2}= & \frac{1}{4 \pi}\left\{\sum _ { i = - N _ { 1 } } ^ { N _ { 1 } } \sum _ { j = - N _ { 2 } } ^ { N _ { 2 } } \sum _ { k = - N _ { 3 } } ^ { N _ { 3 } } \left[\frac{i^{2}+j^{2}-2 k^{2}}{\left(i^{2}+j^{2}+k^{2}\right)^{5 / 2}}\right.\right. \\
& \left.-\frac{3 j^{2}\left(i^{2}+j^{2}-4 k^{2}\right)}{\left(i^{2}+j^{2}+k^{2}\right)^{7 / 2}}\right] \\
& \left.-2 N_{2} \sum_{i=-N_{1}}^{N_{1}} \sum_{k=-N_{3}}^{N_{3}} \frac{i^{2}+N_{2}^{2}-2 k^{2}}{\left(i^{2}+N_{2}^{2}+k^{2}\right)^{5 / 2}}\right\}, \\
\Sigma_{3}= & \frac{1}{4 \pi}\left\{\sum _ { i = - N _ { 1 } } ^ { N _ { 1 } } \sum _ { j = - N _ { 2 } } ^ { N _ { 2 } } \sum _ { k = - N _ { 3 } } ^ { N _ { 3 } } \left[\frac{i^{2}+j^{2}-2 k^{2}}{\left(i^{2}+j^{2}+k^{2}\right)^{5 / 2}}\right.\right. \\
& \left.-\frac{3 k^{2}\left(i^{2}+j^{2}-4 k^{2}\right)}{\left(i^{2}+j^{2}+k^{2}\right)^{7 / 2}}\right] \\
& \left.-2 N_{3} \sum_{i=-N_{1}}^{N_{1}} \sum_{k=-N_{2}}^{N_{2}} \frac{i^{2}+j^{2}-2 N_{3}^{2}}{\left(i^{2}+j^{2}+N_{3}^{2}\right)^{5 / 2}}\right\} .
\end{aligned}
$$

These sums are rapidly convergent, and the values extrapolated to $V / a^{3} \rightarrow \infty$ are independent of the volume shape (i.e., independent of the values of $N_{2} / N_{1}$ and $N_{3} / N_{1}$ ). The results are $\Sigma_{1}=\Sigma_{2}=-0.50493$ and $\Sigma_{3}=1.00986$. Substituting the above expression for $\Lambda_{\xi}$ into Eq. (17) and expanding for small $u_{i j}$ (noting that $n=n^{0}\left(1-u_{k k}\right)$ ), the electrostriction coefficients are obtained by comparing the result with Eq. (2):

$$
\begin{aligned}
& a_{1}=-1.515 \frac{\left(\epsilon^{0}-\epsilon_{c}\right)^{2}}{\epsilon_{c}}, \\
& a_{2}=-\frac{1}{3} \frac{\left(\epsilon^{0}-\epsilon_{c}\right)\left(\epsilon^{0}+2 \epsilon_{c}\right)}{\epsilon_{c}}+0.505 \frac{\left(\epsilon^{0}-\epsilon_{c}\right)^{2}}{\epsilon_{c}} .
\end{aligned}
$$

These results are identical to those obtained in Ref. 10 using the Lorentz cavity approach. In the following section, we show how the same approach may be used to analyze electrostriction of amorphous structures.

\section{Deformation of amorphous microstructures}

The influence of deformation on the microstructure is described by conservation equations for probability distribution functions. ${ }^{23,24}$ For small deformations, the conservation equations for the one and two "particle" distribution functions are

$$
\begin{aligned}
& \mathscr{P}_{1}(\mathbf{r})-\mathscr{P}_{1}^{0}(\mathbf{r})=-\boldsymbol{\nabla} \cdot\left[\mathscr{P}_{1}(\mathbf{r}) \mathbf{U}(\mathbf{r})\right], \\
& \mathscr{P}_{2}\left(\mathbf{r}_{1}, \mathbf{r}_{2}\right)-\mathscr{P}_{2}^{0}\left(\mathbf{r}_{1}, \mathbf{r}_{2}\right)=-\nabla_{1} \cdot\left[\mathscr{P}_{2}\left(\mathbf{r}_{1}, \mathbf{r}_{2}\right) \mathbf{U}\left(\mathbf{r}_{1}\right)\right] \\
&-\boldsymbol{\nabla}_{2} \cdot\left[\mathscr{P}_{2}\left(\mathbf{r}_{1}, \mathbf{r}_{2}\right) \mathbf{U}\left(\mathbf{r}_{2}\right)\right] .
\end{aligned}
$$

$\mathscr{P}_{1}(\mathbf{r})$ and $\mathscr{P}_{2}\left(\mathbf{r}_{1}, \mathbf{r}_{2}\right)$ are the single and pair (unconditional) probability distribution functions, respectively. Again, the superscript ${ }^{0}$ refers to quantities in the nondeformed state, $\mathbf{U}(\mathbf{r})$ is the displacement of an inclusion located initially at $\mathbf{r}$, and $\boldsymbol{\nabla}_{i}$ is the gradient operator taken with respect to position $\mathbf{r}_{i}$. For a statistically homogeneous material, the "single particle" distribution function $\mathscr{P}_{1}(\mathbf{r})$ is equivalent to the inclusion number density,

$$
\mathscr{P}_{1}(\mathbf{r})=n .
$$

A conservation equation for the conditional probability distribution function, $\mathscr{P}\left(\mathbf{r}_{2} \mid \mathbf{r}_{1}\right) \equiv \mathscr{P}\left(\mathbf{r}_{2}, \mathbf{r}_{1}\right) / \mathscr{P}_{1}\left(\mathbf{r}_{1}\right)$, follows from the conservation equations for the one and two inclusion unconditional distribution functions. This relationship, combined with Eq. 28 and the Eq. 29, gives the conservation equation for the conditional probability,

$$
\begin{aligned}
\mathscr{P}\left(\mathbf{r}_{2} \mid \mathbf{r}_{1}\right) \mathscr{P}_{1}^{0}\left(\mathbf{r}_{1}\right)-\mathscr{P}^{0}\left(\mathbf{r}_{2} \mid \mathbf{r}_{1}\right) \mathscr{P}_{1}^{0}\left(\mathbf{r}_{1}\right) \\
=-\left\{\mathscr{P}_{1}^{0}\left(\mathbf{r}_{1}\right)-\nabla_{1} \cdot\left[\mathscr{P}_{1}\left(\mathbf{r}_{1}\right) \mathbf{U}\left(\mathbf{r}_{1}\right)\right]\right\} \\
\cdot\left\{\mathbf{U}\left(\mathbf{r}_{1}\right) \cdot \nabla_{1} \mathscr{P}\left(\mathbf{r}_{2} \mid \mathbf{r}_{1}\right)+\nabla_{2} \cdot\left[\mathscr{P}\left(\mathbf{r}_{2} \mid \mathbf{r}_{1}\right) \mathbf{U}\left(\mathbf{r}_{2}\right)\right]\right\} .
\end{aligned}
$$

For a statistically homogeneous material, the conditional probability depends only upon the separation $\mathbf{r}_{2}-\mathbf{r}_{1}$, in which case $\boldsymbol{\nabla}_{1} \mathscr{P}\left(\mathbf{r}_{2} \mid \mathbf{r}_{1}\right)=-\boldsymbol{\nabla}_{2} \mathscr{P}\left(\mathbf{r}_{2} \mid \mathbf{r}_{1}\right)$. Thus, the conservation equation for the conditional probability distribution function $\mathscr{P}\left(\mathbf{r}_{2} \mid \mathbf{r}_{1}\right)$ for small displacements can be written 


$$
\mathscr{P}\left(\mathbf{r}_{2} \mid \mathbf{r}_{1}\right)=\mathscr{P}^{0}\left(\mathbf{r}_{2} \mid \mathbf{r}_{1}\right)-\nabla_{2} \cdot\left\{\mathscr{P}\left(\mathbf{r}_{2} \mid \mathbf{r}_{1}\right)\left[\mathbf{U}\left(\mathbf{r}_{2}\right)-\mathbf{U}\left(\mathbf{r}_{1}\right)\right]\right\} .
$$

The connection between inclusion displacements $\mathbf{U}\left(\mathbf{r}_{i}\right)$ and the macroscopic deformation depends on the mechanical properties of both the disperse and continuous phases, the inclusion shapes, and any physical or chemical interactions between the inclusions. Determining these relationships is, in general, a challenging problem. We assume here that the inclusions simply follow ambient deformation, i.e., $\mathbf{U}\left(\mathbf{r}_{i}\right)=\mathbf{u}^{\infty}\left(\mathbf{x}=\mathbf{r}_{i}\right)$, where $\mathbf{u}^{\infty}(\mathbf{x})$ is the macroscopic displacement field. This assumption would be appropriate, for example, for a dilute suspension of widely separated rigid inclusions dispersed in a linear elastic continuous phase. For finite concentrations, these displacements would be altered by elastic interactions between the inclusions; ${ }^{27}$ these are neglected in the present analysis. For deformations that are uniform on length scales that are large compared to the inclusion size, an inclusion's displacement can be written

$$
U_{i}=u_{i}^{\infty}(\mathbf{0})+u_{i j} x_{j}+\omega_{i j} x_{j},
$$

where $u_{i j}$ and $\omega_{i j}$ are the deformation and rotation tensors, respectively,

$$
\begin{aligned}
& u_{i j}=\frac{1}{2}\left(\frac{\partial u_{i}^{\infty}}{\partial x_{j}}+\frac{\partial u_{j}^{\infty}}{\partial x_{i}}\right), \\
& \omega_{i j}=\frac{1}{2}\left(\frac{\partial u_{i}^{\infty}}{\partial x_{j}}-\frac{\partial u_{j}^{\infty}}{\partial x_{i}}\right) .
\end{aligned}
$$

For amorphous materials, isotropic prior to deformation, the distribution function $\mathscr{P}_{1}^{0}(\mathbf{r})=\mathscr{P}_{1}^{0}(r)$ depends only on $r=|\mathbf{r}|$, and the conditional pair distribution function $\mathscr{P}^{0}\left(\mathbf{r}_{2} \mid \mathbf{r}_{1}\right)=\mathscr{P}^{0}\left(\left|\mathbf{r}_{12}\right|\right)$ depends only on the scalar separation $\left|\mathbf{r}_{12}\right|$. It follows that $\boldsymbol{\nabla} \cdot\left[\mathscr{P}_{1}^{0}(r) \boldsymbol{\omega} \cdot \mathbf{r}\right]=0$, and $\boldsymbol{\nabla} \cdot\left[\mathscr{P}^{0}\left(\left|\mathbf{r}_{12}\right|\right) \boldsymbol{\omega} \cdot \mathbf{r}_{12}\right]=0$. This expresses the intuitive notion that pure rotation of an isotropic material cannot alter the distribution functions.

The conservation equations for the distribution functions for statistically homogeneous, initially isotropic media can now be written for small deformations $(\|\mathbf{u}\| \ll 1)$ as

$$
\mathscr{P}_{1}(r)=\mathscr{P}_{1}^{0}(r)\left(1-u_{k k}\right),
$$

or

$$
n=n^{0}\left(1-u_{k k}\right)
$$

and

$$
\mathscr{P}(\mathbf{r} \mid \mathbf{0})=\mathscr{P}^{0}(r \mid \mathbf{0})\left(1-u_{k k}\right)-\mathbf{r} \cdot \mathbf{u} \cdot \boldsymbol{\nabla} \mathscr{P}^{0}(r \mid \mathbf{0}) .
$$

These equations differ from Eqs. (28) and (32) only by terms of $O\left(\|\mathbf{u}\|^{2}\right)$. Eq. (37) states that the number density is altered only by a change in volume. The last term in Eq. (38) describes how deformation can produce anisotropy in the conditional pair distribution function.

\section{E. Dielectric tensor}

The quantity $\Lambda_{\xi}$ depends on the function $\mathscr{P}(\mathbf{r} \mid \mathbf{0})$, which has been expressed through the probability density of the nondeformed material $\mathscr{P}^{0}(r \mid 0)$ [Eq. (38)]. Although the integral $\Lambda^{0}$ is conditionally convergent, we proceed by em- ploying the same approach as in Sec. II C. We assume that $\Lambda_{0}$ exists and is known (for example, in terms of $\epsilon^{0}$ ), and express $\Lambda_{\xi}$ in terms of $\Lambda^{0}$.

Average values do not depend on the volume chosen for the averaging procedure, and thus the same control volume $V_{0}$ will be employed in the expression for $\Lambda_{\xi}$ [Eq. (15)] as for $\Lambda^{0}$

$$
\Lambda_{\xi}=\int_{V^{0}}\left(\mathbf{e}_{\xi} \cdot \mathbf{T}(\mathbf{r}) \cdot \mathbf{e}_{\xi}\right) \mathscr{P}(\mathbf{r} \mid \mathbf{0}) d \mathbf{r} .
$$

The influence of deformation on $\Lambda_{\xi}$ is obtained by substituting the pair conservation equation [Eq. (38)] into Eq. (15),

$$
\begin{aligned}
\Lambda_{\xi}= & \int_{V^{0}}\left(\mathbf{e}_{\xi} \cdot \mathbf{T}(\mathbf{r}) \cdot \mathbf{e}_{\xi}\right)\left\{\mathscr{P}^{0}(r \mid \mathbf{0})\left(1-u_{k k}\right)\right. \\
& \left.-\mathbf{r} \cdot \mathbf{u} \cdot \boldsymbol{\nabla}\left[\mathscr{P}^{0}(r \mid \mathbf{0})\right]\right\} d \mathbf{r}=\left(1-u_{k k}\right) \Lambda^{0} \\
& -\int_{V^{0}}\left(\mathbf{e}_{\xi} \cdot \mathbf{T}(\mathbf{r}) \cdot \mathbf{e}_{\xi}\right) \mathbf{r} \cdot \mathbf{u} \cdot \boldsymbol{\nabla}\left[\mathscr{P}^{0}(r \mid \mathbf{0})\right] d \mathbf{r} .
\end{aligned}
$$

Note that Eq. (40) is the integral analog of Eq. (21). The last integral in Eq. (40) depends on the term $\nabla\left[\mathscr{P}^{0}(r \mid \mathbf{0})\right]$; this integral is convergent for many reasonable models of the material microstructure, where $\left|\mathbf{r} \cdot \mathbf{u} \cdot \nabla\left[\mathscr{P}^{0}(r \mid \mathbf{0})\right]\right|$ $\sim o\left(r^{-q}\right)$, with $q>0$. Since $\mathscr{P}^{0}(r \mid \mathbf{0})$ depends only on the magnitude $|\mathbf{r}|=r$, this integral can be calculated directly,

$$
\begin{aligned}
\int_{V^{0}}\left(\mathbf{e}_{\xi}\right. & \left.\cdot \mathbf{T}(\mathbf{r}) \cdot \mathbf{e}_{\xi}\right) \mathbf{r} \cdot \mathbf{u} \cdot \nabla \mathscr{P}^{0}(r \mid \mathbf{0}) d \mathbf{r} \\
= & \frac{1}{4 \epsilon_{0} \epsilon_{c}} \int_{0}^{\pi}\left[\int_{0}^{R(\theta)} \frac{d \mathscr{P}^{0}(r \mid 0)}{d r} d r\left(1-3 \cos ^{2} \theta\right)\right. \\
& \left.\times\left(2 u_{\xi \xi} \cos ^{2} \theta+\left(u_{k k}-u_{\xi \xi}\right) \sin ^{2} \theta\right) \sin \theta\right] d \theta \\
= & -\frac{2 n^{0}}{5 \epsilon_{0} \epsilon_{c}} u_{\xi \xi}+\frac{2 n^{0}}{15 \epsilon_{0} \epsilon_{c}} u_{k k},
\end{aligned}
$$

where the integral $\int_{0}^{R}\left(d \mathscr{P}^{0}(r \mid 0) / d r\right) d r=n^{0}$ for $R \gg v^{1 / 3}(v$ is the inclusion volume) by the definition of $\mathscr{P}^{0}(r \mid 0)$. Substituting Eq. (40) into Eq. (39) gives the final expression for $\Lambda_{\xi}$

$$
\Lambda_{\xi}=\Lambda^{0}\left(1-u_{k k}\right)+\frac{2 n^{0}}{15 \epsilon_{0} \epsilon_{c}}\left(3 u_{\xi \xi}-u_{k k}\right) .
$$

Substituting this into Eq. (17) and expanding for small $u_{\xi \xi}$ and $u_{k k}$ produces

$$
\begin{aligned}
\epsilon_{\xi}= & \epsilon_{c}+\frac{\alpha n^{0}}{\epsilon_{0}\left(1+\alpha \Lambda^{0}\right)}\left[1-\left(\frac{1}{1+\alpha \Lambda^{0}}-\frac{2}{15} \frac{\alpha n^{0}}{\epsilon_{0} \epsilon_{c}\left(1+\alpha \Lambda^{0}\right)}\right)\right. \\
& \left.\times u_{k k}-\frac{2 \alpha n^{0}}{5 \epsilon_{0} \epsilon_{c}\left(1+\alpha \Lambda^{0}\right)} u_{\xi \xi}\right] .
\end{aligned}
$$

Comparing this result with Eq. (9), we obtain the dielectric parameters

$$
a_{1}=-\frac{2}{5}\left(\frac{\alpha n^{0}}{\epsilon_{0}}\right)^{2} \frac{1}{\epsilon_{c}\left(1+\alpha \Lambda^{0}\right)^{2}},
$$




$$
a_{2}=-\frac{\alpha n^{0}}{\epsilon_{0}\left(1+\alpha \Lambda^{0}\right)}\left(\frac{1}{1+\alpha \Lambda^{0}}-\frac{2}{15} \frac{\alpha n^{0}}{\epsilon_{0} \epsilon_{c}\left(1+\alpha \Lambda^{0}\right)}\right) .
$$

At this point, $a_{1}$ and $a_{2}$ are expressed through the microstructure of the nondeformed state via the integral $\Lambda^{0}$. The dielectric constant, $\epsilon^{0}$, is also related to $\Lambda^{0}$, via Eq. (17) applied to the nondeformed state,

$$
\epsilon^{0}=\epsilon_{c}+\frac{\alpha n^{0}}{\epsilon_{0}\left(1+\alpha \Lambda^{0}\right)} .
$$

As discussed above, since $\epsilon^{0}$ is a measurable quantity, $\Lambda^{0}$ may be interpreted simply as a function of $\epsilon^{0}$ without addressing the conditional convergence of $\Lambda^{0}$. Inserting Eq. (46) into Eqs. (44) and (45), we obtain

$$
\begin{aligned}
& a_{1}=-\frac{2}{5} \frac{\left(\epsilon^{0}-\epsilon_{c}\right)^{2}}{\epsilon_{c}}, \\
& a_{2}=-\frac{\left(\epsilon^{0}-\epsilon_{c}\right)^{2} \epsilon_{0}}{\alpha n^{0}}+\frac{2}{15} \frac{\left(\epsilon^{0}-\epsilon_{c}\right)^{2}}{\epsilon_{c}} .
\end{aligned}
$$

These equations satisfy the primary goal of the present model - to express the dielectric parameters in terms of other material properties, specifically, $\epsilon^{0}, \epsilon_{c}, \alpha$, and $n^{0}$. These expressions are useful if all the parameters are known. In cases where $\alpha$ and $n^{0}$ are unknown, these parameters may be eliminated via the reasonable assumption $\Lambda^{0}=-n^{0} / 3 \epsilon_{0} \epsilon_{c}$. This value for $\Lambda^{0}$ is obtained, for example, by the renormalization procedure for disperse spherical inclusions. ${ }^{13,22}$ Inserting this expression into Eq. (46) recovers the ClausiusMossotti equation

$$
\frac{\epsilon^{0}-\epsilon_{c}}{\epsilon^{0}+2 \epsilon_{c}}=\frac{\alpha n^{0}}{3 \epsilon_{0} \epsilon_{c}},
$$

which can be extended to the Debye model if $\alpha$ is replaced by $\alpha+p^{2} / 3 k T$, where $p$ is the permanent dipole moment magnitude. Eq. (49) is equivalent to Maxwell's approximate equation,

$$
\frac{\epsilon^{0}}{\epsilon_{c}}=\frac{1+2 \beta \phi}{1-\beta \phi},
$$

where $\phi \equiv n^{0} v$ is the inclusion volume fraction, and $\beta \equiv \alpha / 3 \epsilon_{0} \epsilon_{c} v$. The dielectric parameters can now be expressed in terms of only $\epsilon^{0}$ and $\epsilon_{c}$,

$$
\begin{aligned}
& a_{1}=-\frac{2}{5} \frac{\left(\epsilon^{0}-\epsilon_{c}\right)^{2}}{\epsilon_{c}}, \\
& a_{2}=-\frac{1}{3} \frac{\left(\epsilon^{0}-\epsilon_{c}\right)\left(\epsilon^{0}+2 \epsilon_{c}\right)}{\epsilon_{c}}+\frac{2}{15} \frac{\left(\epsilon^{0}-\epsilon_{c}\right)^{2}}{\epsilon_{c}} .
\end{aligned}
$$

Eqs. (51) and (52) illustrate that a heterogeneous medium composed of two "nonelectrostrictive" phases is electrostrictive. A solid body treated as polarizable molecules dispersed in a vacuum $\left(\epsilon_{c}=1\right)$ is a common example of a such system.

\section{DISCUSSION AND COMPARISON WITH OTHER MODELS}

The model developed in this article employs traditional assumptions typical of classical dielectric models of isotropic materials (e.g., Clausius-Mossotti, Maxwell, and Debye):

(1) Dipolar polarization is considered (higher-order electrostatic moments are ignored);

(2) the dipole moment of each inclusion is proportional to the local field, $\boldsymbol{\mu}=\alpha \mathbf{E}_{\mathrm{loc}}$;

(3) the material is isotropic; and

(4) each inclusion contributes equivalently to the local field and the total polarization (mean-field assumption).

Additional assumptions were made in order to describe the material behavior for arbitrary deformations, and to relate the composition and microstructure of dielectric solids to the dielectric properties $\epsilon^{0}, a_{1}$, and $a_{2}$ :

(1) The deformation is small;

(2) deformation influences $\mathscr{P}(\mathbf{r} \mid \mathbf{0})$ and $n\left(\equiv \mathscr{P}_{1}(\mathbf{r})\right)$; and

(3) the dipoles displace affinely with the macroscopic deformation.

These assumptions were chosen with the objective of obtaining expressions for $a_{1}$ and $a_{2}$ in terms of measurable properties, but with limited information about the microstructure.

The approach developed in this article can be improved or extended to include other features when more information about the microstructure is available. For example, other polarization mechanisms (e.g., permanent dipole orientation, Maxwell-Wagner polarization, etc.) can be treated, provided that the excess polarization can be expressed in terms of other properties. Measured conditional probability distribution functions may be employed directly in calculations for materials whose microstructures do not satisfy our assumptions. The assumption of affine deformation of the dipoles may be relaxed, for instance, if the inclusions were known to be dispersed in a linearly elastic medium. In this case, the methods described in Ref. 27 can be employed to determine the relative inclusion displacements. Similarly, relative displacements in a viscoelastic continuous phase can be incorporated if a model for the time-dependent rheology of the matrix is specified. The influence of the deformation dependence of $\alpha$ and $\epsilon_{c}$ on electrostriction may also be determined, provided that these dependencies are known. These effects, however, will likely require analyzing polarization in anisotropic media [e.g., if the deformation dependence of the continuous phase permittivity is expressed in the form of Eq. (2)]. Many such features will be material specific, and thus we have neglected them in this preliminary analysis.

Stratton ${ }^{1}$ considered electrostriction of fluids composed of polarizable molecules, whose dielectric properties are described by the Clausius-Mossotti model [Eq. (49) with $\left.\epsilon_{c}=1\right]$. Since fluids can only sustain isotropic deformations, Stratton obtained $a_{1}=0$ and $a_{2}=-(1 / 3)\left(\epsilon^{0}-1\right)\left(\epsilon^{0}+2\right)$. Our formalism describes general anisotropic deformations, but reduces to the same result for isotropic deformation, as expected since the same physical model is employed for the dielectric properties. For $u_{11}=u_{22}=u_{33}=u_{k k} / 3, \epsilon_{\xi}=\epsilon^{0}$ $+\left(a_{1} / 3+a_{2}\right) u_{k k}$, which gives the same deformation- 


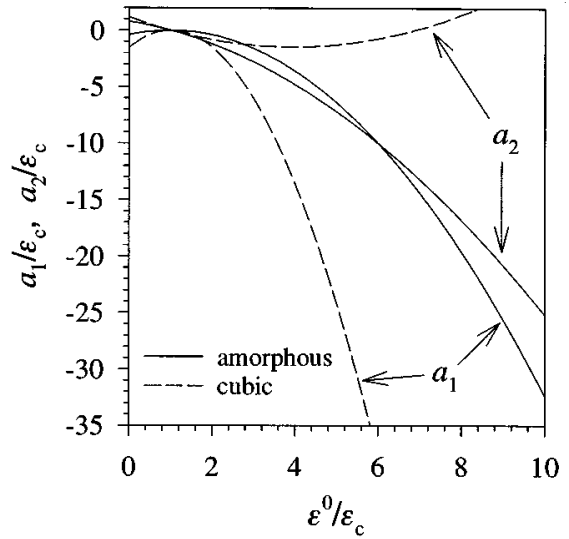

FIG. 3. The parameters $a_{1}$ and $a_{2}$ as functions of $\epsilon^{0} / \epsilon_{c}$ for an amorphous structure and a cubic lattice. The differences between the results for amorphous and cubic structures illustrate the sensitivity of electrostrictive properties to the details of the microstructure. A cubic structure is apparently not a good model for amorphous materials when investigating electrostriction.

dependent dielectric constant predicted by Stratton.

Anderson ${ }^{10}$ considered a material composed of polarizable entities arranged on a cubic lattice in a vacuum, which translate affinely with the macroscopic deformation. The variation in permittivity was attributed only to the changes in the number density and the local field. These assumptions were also employed in the model developed above (with $\left.\epsilon_{c}=1\right)$. However, in Ref. 10, the influence of distant dipoles on the local field was determined with the Lorentz cavity approach. The dielectric parameters can be extracted from the analysis in Ref. 10 and are the same as our results for a cubic lattice [Eqs. (26) and (27)].

The coefficients $a_{1}$ and $a_{2}$ are plotted as a function of $\epsilon^{0} / \epsilon_{c}$ in Fig. 3 for both the amorphous and cubic structures. The two models differ qualitatively and quantitatively. Predictions for amorphous structures give $a_{2}<0$ for all $\epsilon^{0} / \epsilon_{c}>1$, whereas for the cubic structure, $a_{2}>0$ for $\epsilon^{0} / \epsilon_{c}>6.8$. The significant differences between the two predictions suggest that a cubic lattice is not a good model for amorphous structures when studying electrostriction. At small concentrations $(\phi \ll 1)$, predictions give $a_{1} \propto \phi^{2}$ while $a_{2} \propto \phi$. The parameter $a_{1}$ is much smaller in this regime since it describes anisotropic contributions to permittivity tensor, which require at least two-body interactions. The parameter $a_{2}$, on the other hand, describes in part the increase in dielectric constant due to the change in inclusion number density, and thus scales linearly with concentration.

These results illustrate that various physical and structural features affect the magnitudes of $a_{1}$ and $a_{2}$, and that these parameters do not depend on system properties in the same way. Thus, understanding these effects may provide new approaches for synthesizing electrostrictive materials with useful properties, and perhaps suggest new methods for studying the materials' structure.

\section{COMPARISON WITH EXPERIMENTAL DATA}

To properly compare the model developed here with experimental data, Eqs. (51) and (52) [as well as Eqs. (26) and

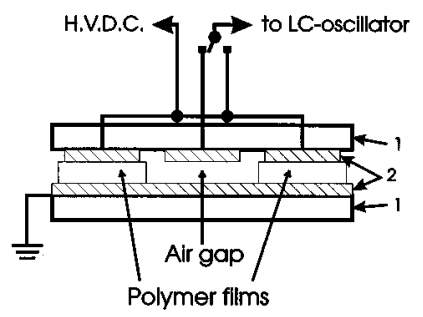

FIG. 4. Schematic diagram of the polymer-air-polymer capacitor assembly. The different layers are: 1-glass slides, 2-electrodes. Two polymer films, obtained by cutting a single film in half, act as spacers for the center air-gap capacitor.

(27)] should be compared with measured values of $a_{1}$ and $a_{2}$ for a variety of different materials with different values of $\epsilon^{0}$. An experimental technique appropriate for obtaining the parameters $a_{1}$ and $a_{2}$ has been described previously. ${ }^{4}$ The technique employs a composite polymer-air-polymer capacitor, illustrated in Fig. 4, which allows for simultaneous measurement of deformation and changes in permittivity of a polymer sample. Two polymer films, obtained by cutting a single film in half, act as a spacer for the center air-gap capacitor. The two outer electrodes are maintained at the same electric potential to insure that an applied electric field equally deforms both films. Thus, when the sample deforms, the change in capacitance of the air-gap capacitor yields the change in the film thickness, while the capacitance change of the polymer-film capacitor reflects both the thickness and permittivity changes. Results from a typical experiment are presented in Fig. 5, where the relative capacitance change of the air-gap capacitor $\left(\delta h / h \approx-\delta C^{\text {air }} / C^{\text {air }}\right)$ and the polymerfilm capacitor $\left(\delta h / h-\delta \epsilon / \epsilon \approx-\delta C^{\text {poly }} / C^{\text {poly }}\right)$ are plotted as

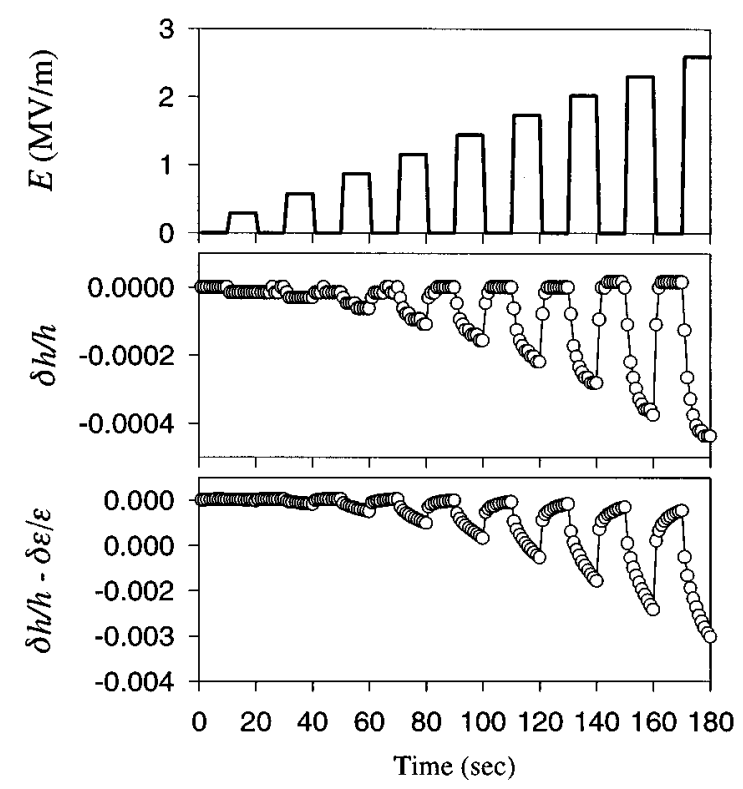

FIG. 5. Capacitance changes for the air-gap capacitor $\left(\delta h / h \approx-\delta C^{\mathrm{air}} / C^{\mathrm{air}}\right)$ and the polymer-film capacitor ( $\left.\delta h / h-\delta \epsilon / \epsilon \approx-\delta C^{\text {poly }} / C^{\text {poly }}\right)$ as functions of time for a square-wave electric field of linearly increasing magnitude. Data shown here are for a Dow 2103-80AE polyurethane film. The capacitance changes are quadratic in the field strength as expected for a purely electrostrictive response. 


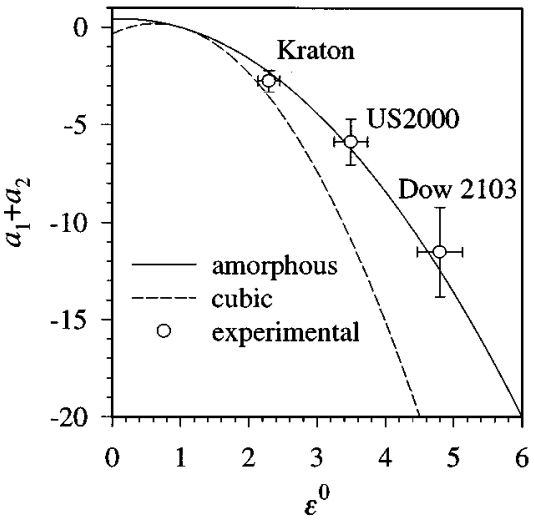

FIG. 6. Comparison of predicted and measured values of $a_{1}+a_{2}$ as a function of $\epsilon^{0}$. Cubic structure, Eqs. (26) and (27); amorphous structures, Eqs. (51) and (52).

functions of time for a square-wave electric field of linearly increasing magnitude. For small uniform deformations, $u_{33} \approx \delta h / h$ and $\delta \epsilon_{33} / u_{33} \approx \delta \epsilon /(\delta h / h)$, and thus the parameters $a_{1}$ and $a_{2}$ may be extracted from the data via Eq. (2). As described in Ref. 4, we use the capacitance changes measured immediately after the field is applied (response averaged over $0.1-0.4 \mathrm{~s}$ ), and then analyze these data as a function of electric field strength.

Tests were performed with three different types of solid block copolymer films. Kraton ( $D-1111$; styrene-isoprenestyrene; Shell) films were solvent cast from toluene. ${ }^{28}$ Dow 2103-80AE (Dow) and US2000 were two microphaseseparated urethane-ether block copolymer films provided by E. Balizer from the Naval Surface Warfare Center in Bethesda, MD. All three films were adhesive. While secured between the glass slides with the electrodes, they were heated to $90^{\circ} \mathrm{C}$ for $1 \mathrm{~h}$. This procedure ensures that the films are constrained and do not slip at the electrode surfaces when deformed. Under this condition, Eq. (2) reduces to $\delta \epsilon_{33}=\left(a_{1}+a_{2}\right) \delta h / h$. Permittivities were measured by General Radio RLC 1689 Digibridge at $100 \mathrm{kHz}$.

These block copolymers can be considered as composites of polarizable domains (or permanent dipole moments) dispersed in soft phases with a smaller dielectric constant, ${ }^{29}$ or as individual molecules or atoms in a vacuum $\left(\epsilon_{c}=1\right)$. Both physical interpretations of the material structure are consistent with our model to a first approximation. In fact, the Clausius-Mossotti model, to which our model reduces in the nondeformed state, has been applied for both cases. ${ }^{7} \mathrm{Nei}-$ ther model, however, accounts for time-dependent polarization, and thus agreement with experimental data at any specific frequency may be only qualitative. To compare model predictions and experimental data, we consider the film materials as collections of polarizable molecules in a vacuum $\left(\epsilon_{c}=1\right)$. Fig. 6 shows the experimental values of $a_{1}+a_{2}$ plotted as a function of $\epsilon^{0}$, along with the predictions for amorphous [Eqs. (51) and (52)] and cubic crystalline [Eqs. (26) and (27)] structures. There is good agreement between the theoretical predictions for amorphous structures and the experimental results for these materials. Since the model development employed simplifying assumptions (e.g., the mean-field approach, neglecting electrostatic multipole interactions, affine displacement of the dipoles, frequency independent parameters), as well as neglected contributions to electrostriction (e.g., the variation in $\alpha$ and $\epsilon_{c}$ with deformation), the agreement illustrated in Fig. 6 may be fortuitous, and results for other materials may not agree as well. Discriminating between possible mechanisms of electrostriction, as well as improving upon the model developed here, requires comparing predictions with much more experimental data. Correlating the extent of agreement with chemical and structural variations may help to illuminate important microscopic phenomena (e.g., effect of deformation on $\alpha$ and $\epsilon_{c}$ ).

\section{CONCLUSION}

We have presented a method for determining the dielectric parameters that describe electrostriction in initially dielectrically isotropic media. This method has been employed to calculate the dielectric parameters for two different types of microstructures: an amorphous solid, which had not been previously addressed, and a cubic lattice, which has been considered in an analysis that employed the Lorentz cavity approach. The model employed in this article is similar, but avoids the Lorentz cavity approach.

Our predictions for electrostriction in amorphous and cubic structures differ significantly. This illustrates the sensitivity of electrostrictive properties to details of the microstructure, and that a cubic structure is not necessarily an appropriate model for amorphous, isotropic materials.

The analysis presented in this article provides a clearer understanding of the microscopic origin of electrostriction. Specifically, the redistribution of dipoles during deformation produces electrostriction in two different ways-through the change in the dipole number density (singlet distribution function), and through the appearance of anisotropy in the microstructure (in the pair distribution function). The parameter $a_{1}$ arises only from induced anisotropy, while $a_{2}$ contains contributions from both effects. Understanding these mechanisms may provide new approaches for synthesizing new electrostrictive materials with prescribed properties, and perhaps suggests new methods for studying material structure. The present model can be extended, in a straightforward manner, to include more complicated features such as nonaffine displacement of inclusions, viscoelastic deformation, and other polarization mechanisms.

Model predictions agree with existing experimental data. Further progress in understanding electrostriction and developing improved models requires measuring $a_{1}$ and $a_{2}$ for various materials and under various conditions (field strength and frequency, deformation frequency, temperature, etc.).

\section{ACKNOWLEDGMENTS}

The authors thank V. Naletova from Moscow State University for helpful comments and E. Balizer from NSWC for materials for this study. This work was supported in part by the Office of Naval Research (Grant No. N00014-94-1-0449) and the National Science Foundation (Grant No. CTS9502276). 
${ }^{1}$ J. A. Stratton, Electromagnetic Theory (McGraw-Hill, New York, 1941).

2 J. F. Nye, Physical Properties of Crystals (Oxford University Press, Oxford, 1976).

${ }^{3}$ W. P. Mason, Physical Acoustics and the Properties of Solids (Van Nostrand, Princeton, 1958).

${ }^{4}$ Y. M. Shkel and D. J. Klingenberg, J. Appl. Phys. 80, 4566 (1996).

${ }^{5}$ L. D. Landau and E. M. Lifshitz, Electrodynamics of Continuous Media (Pergamon, New York, 1984).

${ }^{6}$ The definitions of $a_{1}$ and $a_{2}$ follow those in Ref. 5. Similar parameters defined in Ref. 1, $\tilde{a}_{1}$ and $\tilde{a}_{2}$, are related to those employed here by $\tilde{a}_{1}-\tilde{a}_{2}=a_{1}$ and $\tilde{a}_{2}=a_{2}$. These parameters are often referred to as "electrostriction coefficients," but should be distinguished from the coefficients $\gamma_{i j k \ell}$ that describe the deformation caused by an electric field [Eq. (1)].

${ }^{7}$ C. J. F. Böttcher, Theory of Electric Polarization (Elsevier, New York, 1973).

${ }^{8}$ H. A. Lorentz, Ann. Phys. (N.Y.) 9, 641 (1880); H. A. Lorentz, The Theory of Electrons (Dover, New York, 1952).

${ }^{9}$ C. Kittel, Introduction to Solid State Physics (Wiley, New York, 1971).

${ }^{10}$ R. A. Anderson, Phys. Rev. B 33, 1302 (1986).

${ }^{11}$ G. Kloos, J. Phys. D 28, 1680 (1995).

${ }^{12}$ C. K. Purvis and P. L. Taylor, Phys. Rev. B 26, 4547 (1982).

${ }^{13}$ D. J. Jeffrey, Proc. R. Soc. London, Ser. A 335, 355 (1973).
${ }^{14}$ K. Nakamura and Y. Wada, J. Polym. Sci., Polym. Phys. Ed. 9, 161 (1971).

${ }^{15}$ R. I. Zimmerman, C. Suchicital, and E. Fukada, J. Appl. Polym. Sci. 19, 1373 (1975).

${ }^{16}$ M. Oshiki and E. Fukada, J. Mater. Sci. 10, 1 (1975).

${ }^{17}$ Q. M. Zhang, W. Y. Pan, and L. E. Cross, J. Appl. Phys. 63, 2492 (1988).

${ }^{18}$ D. Morichère, V. Denton, F. Kajzar, P. Robin, Y. Lévy, and M. Dumont, Opt. Commun. 74, 69 (1989).

${ }^{19}$ J. I. Scheinbeim, B. A. Newman, Z. Y. Ma, and J. W. Lee, Polym. Prepr. Am. Chem. Soc. Div. Polym. Chem. 33, 385 (1992).

${ }^{20}$ M. Zhenyi, J. I. Scheinbeim, J. W. Lee, and B. A. Newman, J. Polym. Sci. B: Polym. Phys. 32, 2721 (1994).

${ }^{21}$ K. Elhami, B. Gauthier-Manuel, J. F. Manceau, and F. Bastien, J. Appl. Phys. 77, 3987 (1995).

${ }^{22}$ P. M. Adriani and A. P. Gast, Phys. Fluids 31, 2757 (1988).

${ }^{23}$ W. B. Russel and A. P. Gast, J. Chem. Phys. 84, 1815 (1986).

${ }^{24}$ W. B. Russel, D. A. Saville, and W. R. Schowalter, Colloidal Dispersions (Cambridge University Press, Cambridge, 1989).

${ }^{25}$ Lord Rayleigh, Philos. Mag. 34, 481 (1892).

${ }^{26}$ G. K. Batchelor, J. Fluid Mech. 52, 245 (1972).

${ }^{27}$ N. Phan-Tien and S. Kim, Microstructures in Elastic Media (Oxford University Press, Oxford, 1994).

${ }^{28}$ E. Ding, M.S. thesis, University of Wisconsin-Madison, 1997.

${ }^{29}$ M. A. Valance, Ph.D. thesis, University of Wisconsin-Madison, 1983. 